\title{
Reengineered Helmet Coil for Human Brain Studies at 3 Tesla
}

\author{
WOLFGANG DRIESEL, ${ }^{1}$ HELLMUT MERKLE, ${ }^{2}$ STEFAN HETZER, ${ }^{1}$ THOMAS RIEMER, ${ }^{3}$ \\ STEFAN ZYSSET, ${ }^{1}$ HARALD E. MÖLLER ${ }^{1,4}$ \\ ${ }^{1}$ Max Planck Institute for Human Cognitive and Brain Sciences, D-04103 Leipzig, Germany \\ ${ }^{2}$ National Institute of Neurological Disorders and Stroke, National Institutes of Health, Bethesda, Maryland \\ ${ }^{3}$ Group for Clinical NMR Spectroscopy, Interdisciplinary Center for Clinical Research, University of Leipzig, \\ Leipzig, Germany \\ ${ }^{4}$ Department of Radiology, University Hospital Münster, Münster, Germany
}

\begin{abstract}
We describe the further development of a circularly polarized helmet coil for magnetic resonance imaging (MRI) of the human brain at $3 \mathrm{~T}$. The coil is used in transmit and receive (transceive) mode, a useful alternative over commercially available quadrature headcoils with scanners where phased arrays are unavailable. The coil is simple to build. Its structure is based on an assembly of two coplanar dual-loop coils of the split-circle design, which are arranged in crossed fashion. Both coils circumscribe dome-like the human head. Because of the symmetry of the assembly, a high degree of circularly polarized radiofrequency (RF) is obtained within the brain. Bench and in situ measurements of the RF magnetic field, $\mathbf{B}_{1}$, indicated good axial homogeneity and a moderate gradient along the symmetry axis. Compared to a commercial birdcage coil, the signal-to-noise ratio was increased up to a factor of 1.7 as a result of its superior filling factor. Initial applications in healthy volunteers included anatomical MRI and functional imaging with a cognitive paradigm. @ 2005 Wiley Periodicals, Inc. Concepts Magn Reson Part B (Magn Reson Engineering) 27B: 64-74, 2005*
\end{abstract}

KEY WORDS: helmet coil; high-field imaging; human brain; radiofrequency field homogeneity; specific absorption rate

\section{INTRODUCTION}

Most biomedical magnetic resonance (MR) investigations require a high degree of spatial, temporal, or spectral resolution. To achieve sufficient resolution, a high signal-to-noise ratio (SNR) is of paramount im-

Received 1 February 2005; revised 28 March 2005; accepted 28 March 2005

Correspondence to: Wolfgang Driesel; E-mail: driesel@ cbs.mpg.de; or Hellmut Merkle; E-mail: merkleh@mail.nih.gov.

Concepts in Magnetic Resonance Part B (Magnetic Resonance Engineering), Vol. 27B(1) 64-74 (2005)

Published online in Wiley InterScience (www.interscience.wiley. com). DOI 10.1002/cmr.b.20052

(C) 2005 Wiley Periodicals, Inc. *This article was prepared by a group consisting of both United States government employees and non-United States government employees, and as such, is subject to 17 U.S.C. Sec. 105. portance. This is especially demanding in functional magnetic resonance imaging (FMRI), where subtle signal changes of the order of a few percent or less must be reproducibly identified or in localized spectroscopy where millimolar metabolite concentrations must be accurately quantified in vivo.

It is well known that the design of the radiofrequency (RF) coils is one of the crucial fundamentals for optimizing the SNR in MR (1). Surface coils, consisting in their simplest form of a single circular loop of wire of radius $R$, provide high sensitivity (2). As an example, implanted surface coils enabled ultrahigh resolution FMRI in primates with voxels containing less than 1000 cortical neurons (3). However, the SNR benefit is achieved only at the expense of image homogeneity. Following Chen and Hoult (4), the SNR of a surface coil is maximized at a depth $x$ if 
the coil radius is $R=x / \sqrt{5}$. However, the RF field $\mathbf{B}_{1}$ at $x$ is then only about one fifteenth of that at the surface. Consistently, by comparing surface and whole-volume coils, Edelstein et al. (5) demonstrated that a much higher SNR was achieved by the local coil in peripheral regions, whereas the whole-volume coil was superior in deeper regions. Hence, optimizing the sensitivity over a given region of interest may significantly limit the accessible imaging volume. The issue of limited coverage can be addressed by using an assembly of surface coils (6).

An alternative approach is an anatomically shaped, circularly polarized helmet coil introduced by Merkle et al. (7). It may be considered as an assembly of two crossed surface coil pairs. Previously, this concept was suggested to maximize the filling factor (i.e., the SNR) in the superior brain areas, such as the location of the motor cortex (7-9). For this purpose, the helmet design was short along its longitudinal direction (i.e., the $z$-axis) to avoid loading from more caudal parts. Indeed the substrate for mounting the coil elements was a football helmet. The design has been used successfully for human blood-oxygen level dependent (BOLD) FMRI at $4 \mathrm{~T}(10,11)$ and to image human brain perfusion employing continuous arterial spin labeling (12-14). However, the trade-in was a large $z$-gradient of the RF field $\mathbf{B}_{1}$. To permit a greater variety of FMRI studies, we modified the short design to obtain a more general-purpose transceive coil. High-resolution imaging of the entire brain shows improved SNR compared with a standard birdcage head coil. Additional advantages of the coil described here include stabilization with respect to different loading conditions, easier handling, and more convenient audio-visual paradigm presentation.

\section{DESIGN OF THE COIL}

To maximize the sensitivity while maintaining sufficient RF homogeneity, we engineered the structure with (i) the highest possible symmetry and (ii) structure elements located as close as possible to the head. The shape of the object to be investigated obviously dictates the achievable degree of symmetry of the coil. The brain of a human subject aligned along the main magnet's $z$-axis provides substantial symmetry. Hence, a high degree of circular polarization of the generated RF is obtained in the volume of interest. The design, which circumscribes the whole human head is based on two crossed coplanar dual loop coils arranged coaxially along $z$ while sharing a common circular base in the $x y$ plane (Fig. 1). The circular base contains four distributed capacitors defining four points at halfway between them. The RF is fed (at points $X, X^{\prime}$ and $\left.Y, Y^{\prime}\right)$ at two pairs of spokes. The utilization of a single base is possible because the differential electric potential created halfway is zero.

For better demonstration of the principle of the circuit, we consider a single branch, for example the $X$ spoke pair [Fig. 1(A), left]. Radiofrequency is applied at the top by a tune/match circuit as outlined in Fig. 2. The resulting current splits at the feed-point $X_{P}$ to the circular base and rejoins again from the circular base at the opposite point $X_{P}{ }^{\prime}$. Because of the wellmaintained symmetry, the other two feed-points to the circular base are located at exactly half potential. The same situation applies to the $Y$ spoke pair [Fig. 1(A), right]. The spokes form a dome-like structure above the circular base. Radiofrequency currents in the spokes induce an alternating field, $\mathbf{B}_{1}$, predominantly perpendicular to the static field, $\mathbf{B}_{0}$, and are therefore MR active over the full lengths. Currents in the circular base produce $\mathbf{B}_{1}$ components parallel to $\mathbf{B}_{0}$, which are predominantly MR inactive within the volume of interest.

An interesting aspect of the coil concept is the freedom of tuning. The symmetric arrangement allows independent RF feeding into the structure sharing a common base. Hence, the two pairs of spokes, which are intrinsically decoupled, may be tuned to different resonance frequencies. This can be exploited, for example, for proton-decoupled heteronuclear spectroscopy. The circular base can as well be tuned independently without affecting the other two resonances due to the equal-potential-point connections of the spokes. In the present case, we used the same resonance frequency $(f=125.4 \mathrm{MHz}$, i.e., the ${ }^{1} \mathrm{H}$ Larmor frequency at $2.95 \mathrm{~T}$ ) for all three circuits. To obtain a high degree of circularly polarized $\mathbf{B}_{1}$ within the common sensitive volume of the two loops, $\mathrm{RF}$ was fed $90^{\circ}$ out of phase at points $X-X^{\prime}$ and $Y-Y^{\prime}$.

The spokes and the circular base were made from $35-\mu \mathrm{m}$ thick strips of copper foil. According to Carlson (15), the surface current on a thin foil conductor of width $2 b$ is approximately

$$
j(x)=\frac{I}{2 \pi} \cdot \frac{1}{\sqrt{b^{2}-x^{2}}} \text { for }-b \leq x \leq b,
$$

where $I$ is the total current flowing down the foil. This current distribution has singularities at the edges, that is, the major RF current flows at the edges of the strip. The strip segments of each RF spoke tie their two current-carrying sides to equal potential. With this structural feature, $\mathbf{B}_{1}$ interaction of the two parallel RF current paths is avoided, and the current distribu- 

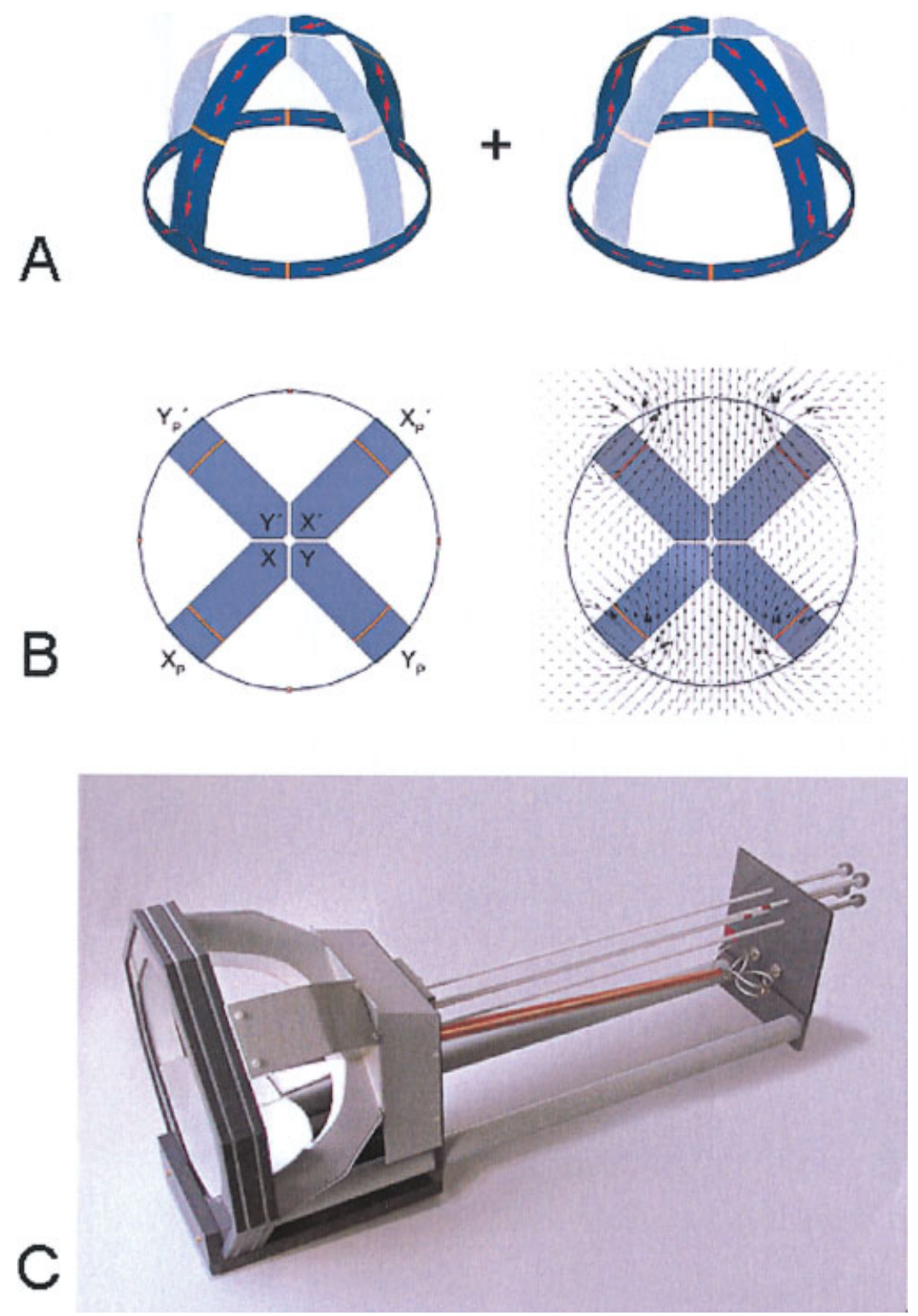

Figure 1 Transceive quadrature helmet coil for investigating the entire human brain. (A) The principle of the helmet coil (9) consists of a combination of two currents that are $90^{\circ}$ out of phase and flow along the two pairs of spokes, $X X_{\mathrm{P}}, X^{\prime} X_{\mathrm{P}}{ }^{\prime}$ (left) and $Y Y_{\mathrm{P}}, Y^{\prime} Y_{\mathrm{P}}{ }^{\prime}$ (right), and a circular base. Positions of the capacitors are indicated in orange. The notation of the feed points $X, X^{\prime}, Y$, and $Y^{\prime}$ from the tune/match circuit and $X_{\mathrm{P}}, X_{\mathrm{P}}{ }^{\prime}, Y_{\mathrm{P}}$, and $Y_{\mathrm{P}}{ }^{\prime}$ to the circular base is defined in (B). The right-hand side in (B) presents a result of a numerical calculation of the $\mathbf{B}_{1}$ field distribution (unloaded coil at phase $\phi=45^{\circ}$ ) in an axial plane viewed from the bottom of the coil. (C) Coil prototype for operation at $3 \mathrm{~T}$. Note that the spokes are arranged at angles $\pm 45^{\circ}$ with respect to the mid-sagittal plane through the human head. This provides more space for additional audiovisual stimulation devices compared with the original helmet coil with spokes at 0 and $90^{\circ}(7)$.

tion is homogeneous over space avoiding hot spots along the spokes.

\section{$B_{1}$ Field Calculations}

To optimize the coil structure for homogeneity within the brain numerical calculations of the RF field dis- tribution were performed. The simulations further provided a starting point for examining potential hot spots and safety distances between head and coil. Assuming a relative permittivity of $\varepsilon_{r}=63.4$ and a conductivity of $\sigma=0.46 \mathrm{~S} / \mathrm{m}(16,17)$ for brain tissue at $125.4 \mathrm{MHz}$ the half wavelength is $\lambda / 2 \approx 15 \mathrm{~cm}$, 


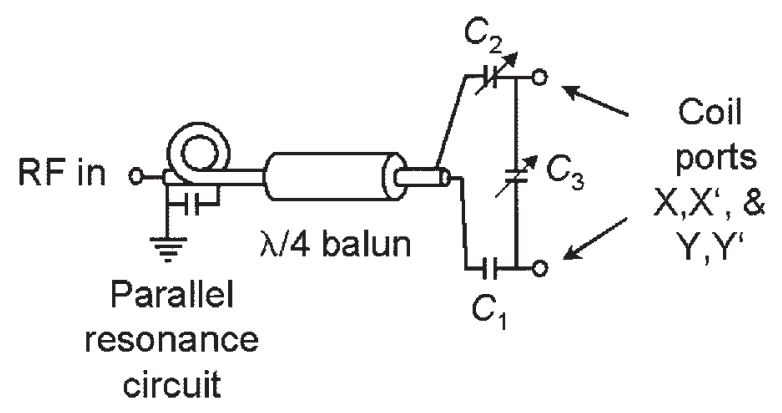

Figure 2 Tuning and matching network of the helmet coil. Two match capacitors, $C_{1}(12 \mathrm{pF})$ and $C_{2}$ (variable), are used to fit the RF to the variable tune capacitor, $C_{3}$.

which is similar to the size of typical structure elements of the object (i.e., the human head). Under such conditions, quasi-static field theory calculations based on Biot-Savart's law represent only a crude approximation (18).

Solving Maxwell's equations with specific boundary conditions yields appropriate results. Numerical calculations of the three-dimensional (3D) field distribution were performed using a personal computer with the commercially available High Frequency Structure Simulator (HFSS 9, Ansoft Corp., Pittsburgh, PA). A 3D parametric design of the helmet coil including the material properties, capacitors, and excitation ports was modelled with the CAD interface of the program package. The quadrature mode of the helmet coil was achieved by assigning the phase offset of the driving voltage source in coil $X$ to $0^{\circ}$ and in coil $Y$ to $90^{\circ}$ [Fig. 1(A)]. Employing the finite element method (16) and adaptive meshing, HFSS solves Maxwell's equations with given boundary conditions in the frequency domain $(19,20)$. Calculations are performed for sinusoidal steady-state fields, and a complex phasor representation (denoted by a script font) is used for the representation of electric and magnetic field quantities, such as $\mathscr{B}_{1}(x, y, z)=\mathbf{B}_{1}(x$, $y, z) \exp [i \phi(x, y, z)]$. The appropriate physical quantity in the time domain is, hence, obtained after multiplication by $\exp (i \omega t)$ and extraction of the real part, $\mathbf{B}_{1}(x, y, z, t)=\operatorname{Re}\left\{\mathscr{B}_{1}(x, y, z) \exp (i \omega t)\right\}$. Here, $\omega$ is the angular frequency of the current, $\phi$ is its phase, $t$ is time, and $i=\sqrt{-1}$. Because we are only interested in the transverse components of the RF, the $z$-component of the phasor is subsequently set to zero because it does not interact with the MR system. Assuming the nuclear spin precession to be in the positive (i.e., counterclockwise) direction, only the positive circularly polarized component of the transmitting field contributes to the excitation of the spin system. It has been outlined by Hoult (21) that for an arbitrary complex function, which can be expressed as $(a+i b)$ $\exp (i \omega t)$, the quantities $a$ and $b$ represent the length of vectors along the $\tilde{x}$ and $\tilde{y}$ directions of a positively rotating frame of reference (denoted by tildes). The complex coefficient $(a+i b)$ has the same structure as the output from HFSS after projection onto the transverse plane; hence, the components $\tilde{B}_{1 x}$ and $\tilde{B}_{1 y}$ of the RF field in the rotating frame (and thereby the interacting RF field amplitude, $\tilde{B}_{1 x y}$ ) are easily obtained from the real and imaginary parts of the phasor.

As a first impression of the $\tilde{B}_{1 x y}$ field homogeneity inside the helmet coil, the right-hand side of Fig. 1(B) shows numerical results computed under unloaded conditions taking a phase angle of $45^{\circ}$. A subsequently performed phase animation demonstrated a nearly perfect circular polarization in the region of the head with a high degree of homogeneity in quadrature mode. For optimizing the design, numerical calculations were then performed with variation of the length of the helmet coil and of the width of the copper foil on the spokes. A specific purpose was to minimize the RF field gradient along the $z$-axis to achieve full coverage of the human brain. If the width of the copper foil was too narrow, an unwanted hot spot resulted in the upper area of the helmet, where the RF is fed to the spokes. In the case that the foil became too wide, $\tilde{B}_{1 x y}$ collapsed in the upper part of the head leading to a loss in sensitivity. Likewise, a short helmet did not achieve sufficient $\tilde{B}_{1 x y}$ throughout the brain. By contrast, for a very long helmet, the coil volume became undesirably large, reducing the filling factor and thus the SNR. Good results for our experimental conditions were obtained with $50-\mathrm{mm}$ wide copper strips and an overall helmet length of $200 \mathrm{~mm}$, which was subsequently used for the construction of the coil. This provides considerably more space as compared to the old design that had a length of 130 $\mathrm{mm}$ and an elliptical base with diameters of 180 and $230 \mathrm{~mm}$.

Using this geometry, further calculations were performed to investigate the RF field distribution for different loading conditions. Figure 3(A,B) shows the magnitude of the $\mathbf{B}_{1}$ field in the $x y$ - and $x z$-planes inside the helmet coil, loaded with a cylindrical gel phantom of $20-\mathrm{cm}$ diameter and $23-\mathrm{cm}$ length. A mixture of $20 \mathrm{~g} / \mathrm{L}$ agarose, $2 \mathrm{~g} / \mathrm{L} \mathrm{NaCl}$, and $1.56 \mathrm{~g} / \mathrm{L}$ $\mathrm{CuSO}_{4} \cdot 5 \mathrm{H}_{2} \mathrm{O}(22)$ was used to mimic dielectric properties of tissue. Experimental values at $40^{\circ} \mathrm{C}$ and $100 \mathrm{MHz}$ for this composition of $\varepsilon_{r}=76$ and $\sigma=$ $0.33 \mathrm{~S} / \mathrm{m}$ determined at PTB Berlin (Seifert F. personal communication) were used in the calculations. The achieved $\tilde{B}_{1 x y}$ amplitude and homogeneity within the phantom were sufficient for in vivo imaging up to a depth of $20 \mathrm{~cm}$ from the top of the coil. 

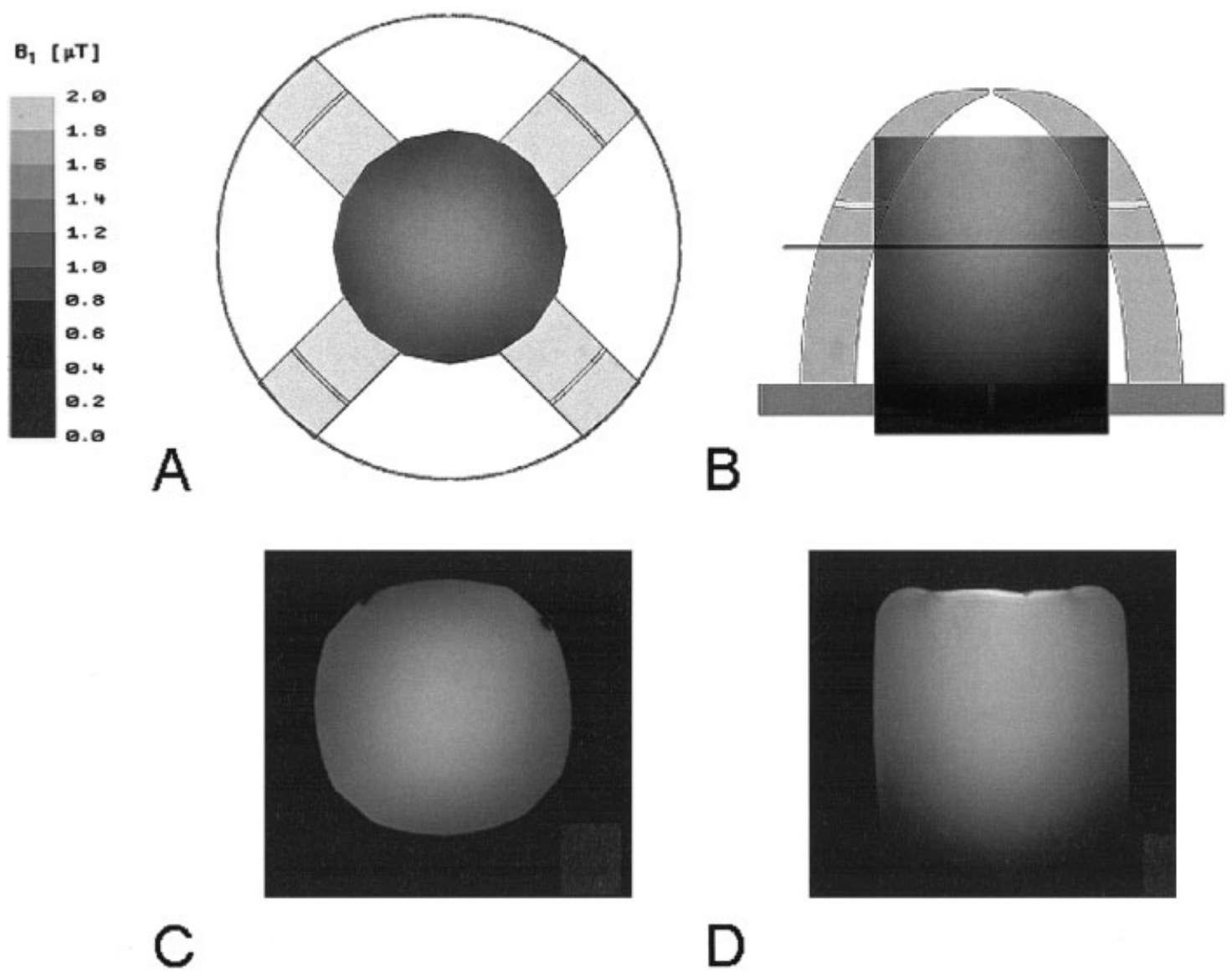

Figure 3 (A) Axial and (B) sagittal $\tilde{B}_{1 x y}$ distribution obtained from numerical simulations of the coil loaded with an agarose gel phantom. The horizontal line in (B) indicates the level of the axial slice. (C) Axial and (D) sagittal experimental MDEFT images obtained at $3 \mathrm{~T}$ with an agarose gel phantom showing good homogeneity up to a depth of approximately $20 \mathrm{~cm}$.

Similar simulation results were obtained with an unstructured human head model (supplied with the software package) and average brain dielectric properties as given above (images not shown). Additional computations of the specific absorption rate (SAR) for each mesh point were performed from the electric field, E, according to (16)

$$
\operatorname{SAR}=\frac{\sigma|\mathbf{E}|^{2}}{2 \rho}
$$

where $\rho$ is the tissue density. A continuous-wave (CW) transmit power of $5 \mathrm{~W}$ (maximum $\mathrm{CW}$ voltage level encoded in the coil file) was assumed to be applied to the coil. Both the maximum SAR averaged over any $1 \mathrm{~g}$ of tissue ("local SAR") as shown in Fig. 4 and the average SAR over the entire head were well below the present limits.

\section{Technical Realization}

A custom-built, dome-like helmet structure [Fig. $1(\mathrm{C})]$ of $275-\mathrm{mm}$ inner diameter at the circular base was utilized as mounting base for all self-sticking copper strips (thickness $35 \mu \mathrm{m}$; Parker Chomerics, Marlow, UK). The coil was finally mounted on a polyvinyl chloride (PVC) substructure that fits onto the patient table of the MR scanner. Each spoke was broken along its lengths by a capacitance of $22 \mathrm{pF}$ for wavelength shortening (to minimize phase shifts along the wires), for tuning the resonance frequency. To improve the dielectric strength, two parallel assemblies of three serially connected $33-\mathrm{pF}$ capacitors were used to yield $22 \mathrm{pF}$. Low inductance of the spokes appeared favorable to achieve a high current at a low voltage corresponding to a high magnetic field but a low electric field. A low foil inductance requires large values for the tuning capacitance according to 

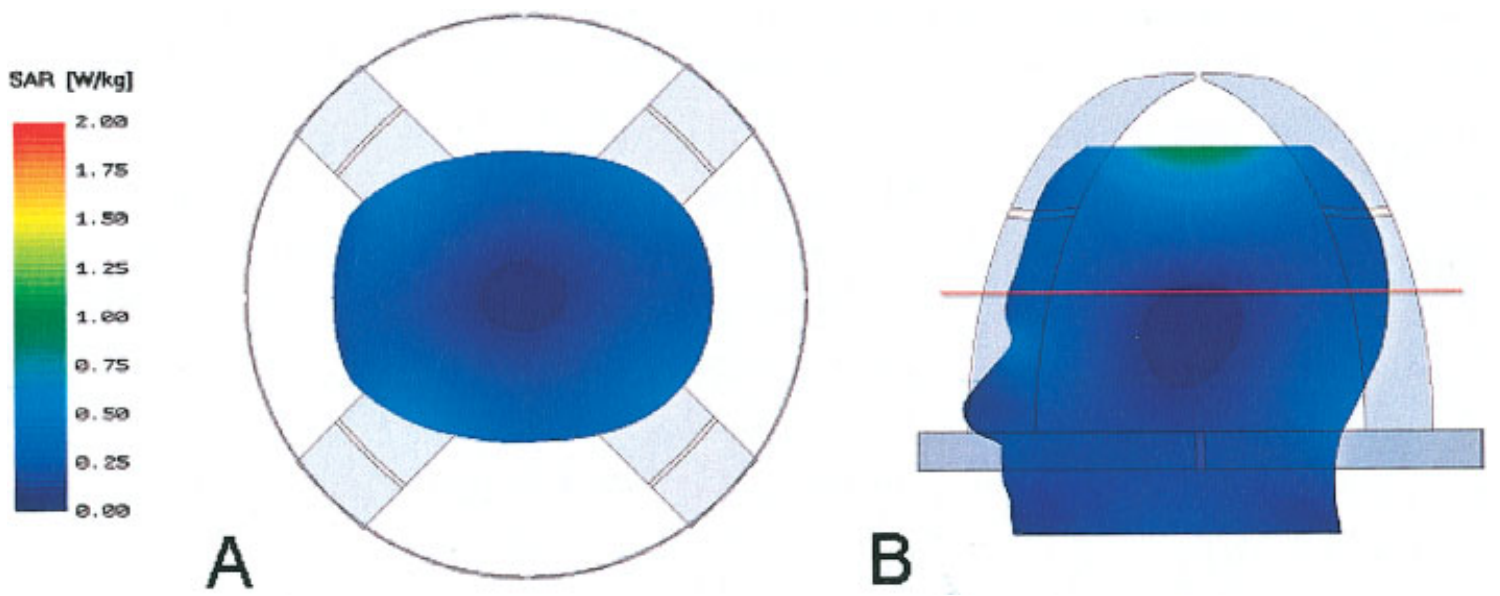

Figure 4 Local SAR levels in a (A) axial and (B) sagittal slice obtained from numerical simulations of the coil loaded with a human head model. The horizontal line in (B) indicates the level of the axial slice.

Thomson's equation. This is also favorable as it minimizes the detuning effect due to the load. The circular base was made from 20-mm wide copper foil. Four capacitors of $8 \mathrm{pF}$ (made from three serially connected capacitors of 27, 27, and $19.2 \mathrm{pF}$ ) were added symmetrically in line for RF wavelength shortening. All fixed capacitors were nonmagnetic type ceramic chips (TEMEX, Sevres, France). On resonance and at a maximum transmit pulse peak power of $5 \mathrm{~kW}$, the voltages at the capacitors are approximately 2.6 and $3.6 \mathrm{kV}$ for the spokes and the circular base, respectively.

Electric interactions between coil and tissue or ground lead to transmission power losses and it lowers the SNR in the case of reception. To minimize this coupling, we utilized an almost perfectly balanced coil design with respect to the sample and ground. To reduce electric stray fields across the sample or to earth, we used the concept of distributed capacitors (23) and a balanced coil design with respect to earth. Two match capacitors $\left(C_{1}=12 \mathrm{pF}\right.$, fixed; $C_{2}=0.5$ $\ldots 25 \mathrm{pF}$, variable) were used to fit the RF to the tune capacitor $\left(C_{3}=0.5 \ldots 25 \mathrm{pF}\right.$, variable) as shown in Fig. 2. The connections from the coil to the tune and match capacitors were as short as possible because this inductance leads to an unwanted magnetic field. The variable capacitors were nonmagnetic trimmer capacitors (Voltronics Inc., Denville, NJ). On resonance, the voltages at $5-\mathrm{kW}$ pulse power at the match and tune capacitors were approximately 1.2 and 1.8 $\mathrm{kV}$, respectively.

To fit the unbalanced coaxial cable to the balanced load of the helmet coil, a quarter-wavelength balun was used. The balun was made from a copper sleeve of $13-\mathrm{mm}$ diameter and placed over the semi-rigid transmission line with a diameter of $3.6 \mathrm{~mm}$. This sleeve, together with the outer jacket of the semi-rigid transmission line, formed a shortened quarter-wave line tuned to the resonance frequency of the coil. The diameter ratio between sleeve and outer jacket of the semi-rigid cable of about 3.6 caused a coaxial-line impedance of $77 \Omega$ and minimized losses in the coaxial line. The impedance looking into the open end of this line was greater than $100 \mathrm{k} \Omega$. The end of the outer conductor of the semi-rigid cable was isolated from the part of the line below the sleeve. This reduced losses caused by currents on the shield of the semi-rigid cable. The balun had no effect on the coil impedance at resonance. However, it added inductive shunt reactance at lower and capacitive shunt reactance at higher frequencies than the quarter-wave resonance frequency. In addition to the balun, we used a parallel resonance circuit for further suppression of standing waves on the outer jacket of the semi-rigid cable. The trap circuit was constructed by bending the semi-rigid transmission outside the balun to a single loop of $60-\mathrm{mm}$ inner diameter. A capacitance was soldered in parallel to his loop inductor in order to form a RF trap tuned to $125.4 \mathrm{MHz}$. This feature in combination with the balun suppressed all parasitic resonances. Isolation of $-16 \mathrm{~dB}$ between both channels was achieved, which was sufficient for good performance of the quadrature coil.

\section{RESULTS AND DISCUSSION}

\section{Dielectric and Magnetic Losses}

An HP 8712B RF-network analyzer (Hewlett-Packard, Palo Alto, CA) was used to study the frequency 
Table 1 Experimental Quality Factor, $Q$, and Frequency Shift, $\delta f$, Measured for Different Loading Conditions

\begin{tabular}{lcccr}
\hline & & \multicolumn{2}{c}{ Loaded Coil } \\
\cline { 3 - 5 } Coil Loop & Unloaded Coil & \multicolumn{2}{c}{ Distilled Water } & Saline Solution \\
\cline { 3 - 5 } & $Q$ & $Q$ & $\delta f$ & $Q$ \\
$Y\left(0^{\circ}\right)$ & 83.47 & 75.28 & $1.214 \mathrm{MHz}$ & 10.27 \\
\hline$\left(90^{\circ}\right)$ & 86.35 & 77.24 & $1.109 \mathrm{MHz}$ & 10.18 \\
\hline
\end{tabular}

dependence of the power reflected from the coil. To determine the contributions of the various loss mechanisms, the quality factor $(Q)$ was investigated for different loading conditions: unloaded, loaded with distilled water, and loaded with saline solution $(0.9 \%$ $\mathrm{NaCl}$ ). Results are summarized in Table 1. The drop in $Q$ upon loading with distilled water, which has a high dielectric constant and a low conductivity, provides a measure of dielectric losses arising from dissipation of RF power due to displacement currents in the tissue $(24,25)$. Because of the balanced coil design, dielectric losses remained small. Additionally, the frequency shift, $\delta f$, upon loading the coil was small. Magnetic losses arise from the additional dissipation of RF power due to eddy currents induced by the RF field in the tissue $(24,25)$. We found for this particular coil that losses from the magnetic interaction within the sample dominate.

\section{Measurements with an Electrically Shielded Pick-up Coil}

For bench measurements of RF magnetic field strength and homogeneity, a pick-up coil was constructed (26), which consisted of a short circuited single loop of coaxial cable (60-mm diameter). The induced current was directed to the network analyzer (50- $\Omega$ input resistance) via a toroidal transformer (15 windings). Additionally, the shield of the coaxial cable was symmetrically broken on the opposite side of the transformer box to block the influence of conservative electric fields on the signal (27). A mechanical sliding device allowed the pick-up coil to be shifted within the helmet coil with an accuracy of about \pm 1 $\mathrm{mm}$. Qualitatively, the shape of the decay of the field strength, $\tilde{B}_{1 x y}$, measured with the pick-up coil along the $z$-axis agreed well with the result from the theoretical simulation of the RF distribution. To compare the experimental and the numerical results directly, the power on the HFSS excitation channel was scaled to achieve an identical level of the RF field as observed experimentally (Fig. 5).

\section{In Situ Experiments on Phantoms}

All measurements were performed at $125.4 \mathrm{MHz}$ using a 3-T MedSpec 30/100 system (Bruker BioSpec, Ettlingen, Germany). For phantom studies, a polyethylene bottle (20-cm diameter, 23-cm length) filled with agarose gel was centered in the coil. To investigate the image quality inside the phantom, a set of modified driven equilibrium Fourier transform (MDEFT) images $(28,29)$ was recorded in sagittal, coronal, and axial slice orientation [flip angle $(\alpha) 20^{\circ}$, repetition time $\left(T_{\mathrm{R}}\right) 10 \mathrm{~ms}$, echo time $\left(T_{\mathrm{E}}\right) 5 \mathrm{~ms}$, inversion time $\left(T_{\mathrm{I}}\right) 650 \mathrm{~ms}$, field of view (FOV) $25 \mathrm{~cm}$, slice thickness $8 \mathrm{~mm}$, matrix $256 \times$ 256; Fig. 3(C,D)]. For comparison, a second series of images was acquired with the coil connected to the RF hybrid in incorrect Larmor rotation sense (images not shown). The intensity ratio of 55:1 obtained from both sets of images is a direct mea-

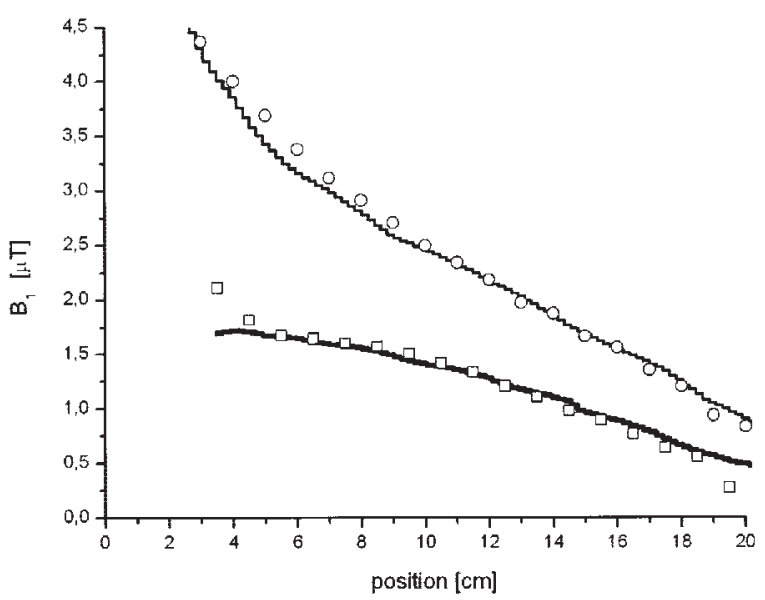

Figure 5 Radiofrequency field amplitude, $\tilde{B}_{1 x y}$, along the symmetry axis of the unloaded helmet coil measured with a pick-up coil $(\bigcirc)$ and in a cylindrical agarose gel phantom recorded with an axial spin-echo sequence (slice thickness 5 $\mathrm{mm}$, inter-slice distance $10 \mathrm{~mm}$ ) and a double-angle approach $(\square)$. Besides the experimental data numerical results based on HFSS calculations are also shown as solid lines (thick line: unloaded coil; thin line: coil loaded with an agarose gel phantom). 
sure of the degree of circular polarization. Approximately $97 \%$ circular polarization was obtained, whereas the remaining portion of the RF was linearly or elliptically polarized.

For further quantitative assessment of the coil efficiency, the amplitude $\tilde{B}_{1 x y}$ was investigated by taking advantage of the principle of reciprocity $(1,21)$. The $\tilde{B}_{1 x y}$ distribution was measured with a doubleangle approach utilizing a simple spin-echo sequence (30). Briefly, effects from longitudinal relaxation can be ignored if the repetition time is sufficiently long $\left(T_{R} \geq 5 T_{1}\right)$. Disregarding transverse coherences and spin interactions, the intensity of the spin echo recorded from position $\mathbf{r}$ within a homogeneous sample with arbitrary flip angles $\alpha$ and $\beta$ for the excitation and refocusing pulse, respectively, is (31)

$$
S(\mathbf{r})=\kappa(\mathbf{r}) \sin \alpha(\mathbf{r}) \sin ^{2} \frac{\beta(\mathbf{r})}{2} e^{-T_{E} / T_{2}}
$$

The proportionality constant $\kappa$ summarizes all further contributions from the receiving system. Two sets of images were acquired with different excitation angles ( $\alpha_{1}=\alpha$ and $\alpha_{2}=2 \alpha$ and otherwise identical sequence parameters to compute $\tilde{B}_{1 x y}(\mathbf{r})$ from the ratio of the echo amplitudes according to

$$
\gamma \tilde{\beta}_{1 x y}(\mathbf{r}) \tau_{p}=\alpha(\mathbf{r})=\arccos \frac{S_{2}(\mathbf{r})}{2 S_{1}(\mathbf{r})}
$$

In Eq. [4], $\gamma$ denotes the gyromagnetic ratio and $\tau_{p}$ the excitation pulse length. Results are given in Fig. 5 along with corresponding simulation data. Within the experimental accuracy, the qualitative agreement with the bench $\tilde{B}_{1 x y}$ measurements using the pick-up coil was found to be excellent. In axial slices, the flip-angle variation was less than $15 \%$ in plane [cf. Fig. 3(C)]. Along the z-axis, there was a moderate $\tilde{B}_{1 x y}$ gradient indicated by a $50 \%$ drop in $\alpha$ over a distance of approximately $10 \mathrm{~cm}$. This gradient is roughly half of that of the original helmet coil and uncritical for imaging deeper regions human brain, as indicated by the persistent signal intensity on the sagittal slice toward the bottom of the phantom shown in Fig. 3(D).

\section{In Vivo Studies}

After certification according to the European Directive for Medical Devices, initial in vivo applications of the new helmet coil included 2D and 3D anatomical imaging with different tissue contrast and FMRI based on the BOLD contrast (32). For this purpose, a total of 14 healthy volunteers (male 7, female 7, age 23-34 years) were investigated after informed written consent had been obtained. The MR procedures had been approved by the Ethics Committee of the University of Leipzig. $T_{1}$-weighted $2 \mathrm{D}$ imaging was performed using a multislice MDEFT sequence $\left(T_{R} 1.3 \mathrm{~s}, T_{E} 9.2 \mathrm{~ms}, T_{I}\right.$ $650 \mathrm{~ms}$, acquisition matrix $256 \times 252$ ). As a special feature, one line of $\mathbf{k}$-space data was obtained from each slice after a single nonselective inversion pulse, which yields a substantial reduction in the amount of RF power required (33). A rapid acquisition with relaxation enhancement (RARE) technique (34) was used for $T_{2}$-weighted $2 \mathrm{D}$ imaging $\left(T_{R} 8.5 \mathrm{~s}\right.$, effective $T_{E} 80 \mathrm{~ms}$, RARE factor 16 , matrix $512 \times 512$ ). The slice orientation (FOV $25 \times 25 \mathrm{~cm}^{2}$, thickness $5 \mathrm{~mm}$, spacing $2 \mathrm{~mm}$ ) was either axial (parallel to the bicommissural plane) or coronal. Whole-head $T_{1}$-weighted 3D MDEFT images (29) were obtained with 128 sagittal slices, $1.5-\mathrm{mm}$ thickness, FOV $25 \times 25 \times 19 \mathrm{~cm}^{3}$, and $256 \times 256$ matrix for image registration. Examples of $T_{1}$ - and $T_{2}$-weighted images are given in Fig. 6 . Whereas only the superior parts of the brain had been visible at sufficient SNR with the original helmet coil, the new design permits high-quality imaging of the entire head. When compared with a commercial quadrature birdcage coil (Bruker BioSpec, Ettlingen, Germany), an SNR gain of up to 1.7, depending on position, is achieved with this design.

For the FMRI experiments, 24 axial slices (FOV $19.2 \mathrm{~cm}$, thickness $4 \mathrm{~mm}$, spacing $1 \mathrm{~mm}$, matrix $64 \times$ 64) covering the whole head were acquired using a single-shot, gradient-recalled echo planar imaging (35) sequence ( $\left.\alpha 40^{\circ}, T_{R} 2 \mathrm{~s}, T_{E} 30 \mathrm{~ms}\right)$. An adapted version of the Stroop color-word interference task $(36,37)$ was used as a cognitive paradigm. The task, which was presented using a single-trial design, has been described in detail elsewhere $(37,38)$. Two functional runs with 363 time steps each were measured. Data were analyzed using LIPSIA (39) with preprocessing steps (motion correction, acquisitiontime compensation, temporal highpass filtering, spatial gaussian filter) on the functional data and statistical analysis with event-related design (randomeffect model). The results of the experiment are shown in Fig. 7. Significant activations were found at the left inferior frontal junction (IFJ), along the left intraparietal sulcus (IPS), in the presupplementary motor area (pre-SMA), and in the left fusiform gyrus (FG), corresponding well with previous studies (37, 38 ) using the standard birdcage head coil. 


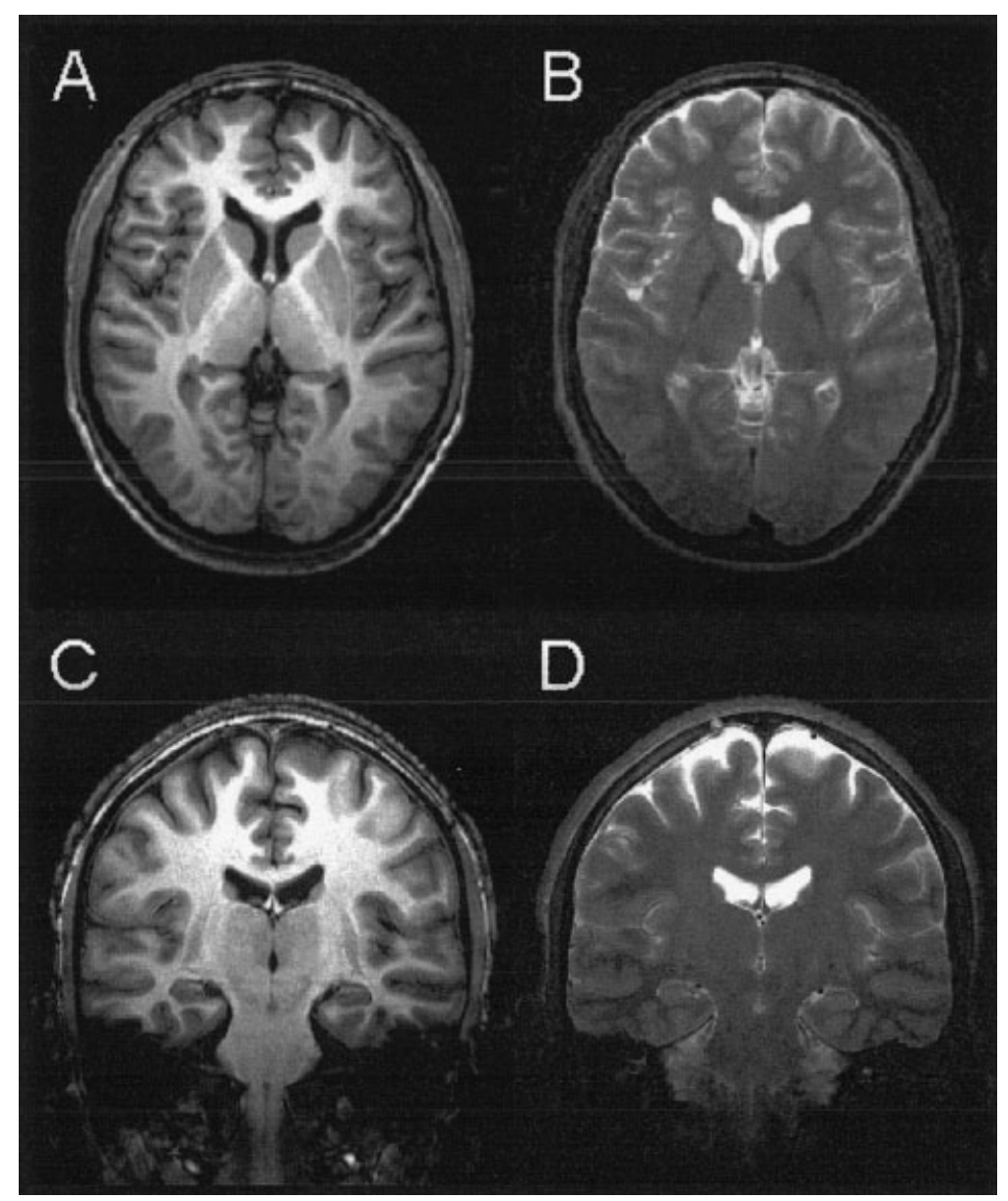

Figure 6 Examples of axial (A,B) and coronal (C,D) $T_{1}$-weighted MDEFT (A,C) and $T_{2}$-weigthed RARE $(\mathrm{B}, \mathrm{D})$ images acquired in a normal human volunteer at $3 \mathrm{~T}$. The moderate $\tilde{B}_{1 x y}$ gradient along the $z$-axis is uncritical for imaging deeper-lying structures of the central nervous system; hence imaging of the entire brain is possible with good tissue contrast.

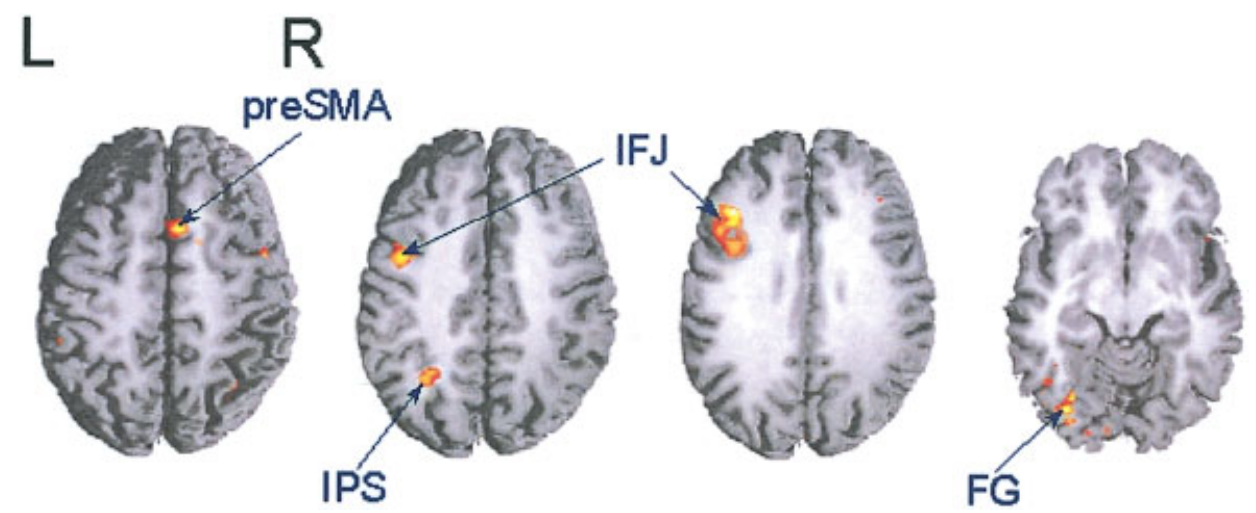

Figure 7 Top view of four axial slices showing the major activations of the Stroop color-word interference task (z-maps). The activations in the presupplementary motor area (pre-SMA), at the left inferior frontal junction (IFJ), along the left intraparietal sulcus (IPS), and in the left fusiform gyrus (FG) represent the contrast between the incongruent and the neutral condition. 


\section{SUMMARY}

The experiments demonstrate that the new helmet coil permits investigations of the entire brain with good tissue contrast. The modified design provides sufficient space for use of additional audiovisual stimulation devices, and appears well suited for anatomical, functional, and metabolic studies at $3 \mathrm{~T}$ or above.

\section{ACKNOWLEDGMENTS}

Valuable support by Toralf Mildner and technical assistance by Manfred Weder are gratefully acknowledged. The authors thank D. Yves von Cramon, David G. Norris, and Margret Hund-Georgiadis for helpful discussions, and Davin Crawford and Markus Laudin from Ansoft Germany for an introduction into HFSS 9. Charlie Jeung from Ansoft kindly provided the head model and Frank Seifert from the PhysikalischTechnische Bundesanstalt Berlin dielectric measurements.

\section{REFERENCES}

1. Hoult DI, Richards RE. 1976. The signal-to-noise ratio of the nuclear magnetic resonance experiment. J Magn Reson 24:71-85.

2. Ackerman JJH, Grove TH, Wong GG, Gadian DG, Radda GK. 1980. Mapping of metabolites in whole animals by ${ }^{31} \mathrm{P}$ NMR using surface coils. Nature 283: $167-170$.

3. Logothetis NK, Merkle H, Augath M, Trinath T, Ugurbil K. 2002. Ultra high-resolution fMRI in monkeys with implanted RF coils. Neuron 35:227-242.

4. Chen CN, Hoult DI. 1989. Biomedical magnetic resonance technology. Bristol: Adam Hilger 340 p.

5. Edelstein WA, Foster TH, Schenck JF. 1985. The relative sensitivity of surface coils to deep lying tissues. In: Society of Magnetic Resonance in Medicine, 4th Annual Meeting, San Francisco, p 964-965.

6. Roemer PB, Edelstein WA, Hayes CE, Souza SP, Mueller OM. 1990. The NMR phased array. Magn Reson Med 16:192-225.

7. Merkle H, Garwood M, Ugurbil K. 1993. Dedicated circularly polarized surface coil assemblies for brain studies at 4 T. In: Proceedings of the Society of Magnetic Resonance in Medicine, 12th Annual Meeting, New York, p 1358.

8. Merkle H, Driesel W, Andersen P, Adriany G, Ugurbil K, Garwood M. 2000. Circular polarized RF helmet coil for brain studies at 7 tesla. In: Proceedings of the International Society for Magnetic Resonance in Medicine, 8th Annual Meeting, Denver, p 565.

9. Silva CA, Merkle H. 2003. Hardware considerations for functional magnetic resonance imaging. Concepts Magn Reson A 16:35-49.

10. Kim SG, Ashe J, Hendrich K, Ellermann JM, Merkle H, Ugurbil K, Georgopoulos AP. 1993. Functional magnetic resonance imaging of motor cortex: Hemispheric asymmetry and handedness. Science 261:615-617.

11. Kim SG, Ashe J, Georgopoulos AP, Merkle H, Ellermann JM, Menon RS, Ogawa S, Ugurbil K. 1993. Functional imaging of human motor cortex at high magnetic field. J Neurophysiol 69:297-302.

12. Trampel R, Mildner T, Goerke U, Schaefer A, Driesel W, Norris DG. 2002. Continuous arterial spin labeling using a local magnetic field gradient coil. Magn Reson Med 48:543-546.

13. Mildner T, Trampel R, Möller HE, Schäfer A, Wiggins CJ, Norris DG. 2003. Functional perfusion imaging using continuous arterial spin labeling with separate labeling and imaging coils at $3 \mathrm{~T}$. Magn Reson Med 49:791-795.

14. Mildner T, Möller HE, Driesel W, Norris DG, Trampel R. 2005. Continuous arterial spin labeling at the human carotid artery: The influence of transit times. NMR Biomed 18:19-23.

15. Carlson JW. 1986. Currents and fields of thin conductors in rf saddle coils. Magn Reson Med 3:778-790.

16. Jin J. 1998. Electromagnetic Analysis and Design in Magnetic Resonance Imaging. Boca Raton: CRC Press; $282 \mathrm{p}$.

17. Federal Communications Commission. Tissue dielectric properties. http://www.fcc.gov/fcc-bin/dielec.sh.

18. Durney CH. 1992. Interactions between electromagnetic fields and biological systems. Ann New York Acad Sci 649:19-34.

19. Lee JF, Sun DK, Cendes ZJ. 1991. Full-wave analysis of dielectric waveguides using tangential vector finite elements. IEEE Trans Microwave Theory Tech 39: 1262-1271.

20. Lee JF, Sun DK, Cences ZJ. 1991. Tangential vector finite elements for electromagnetic field computation. IEEE Trans Magn 27:4032-4035.

21. Hoult DI. 2000. The principle of reciprocity in signal strength calculations-a mathematical guide. Concepts Magn Reson 12:173-187.

22. Hoffmann W, Seifert F, Wlodarczyk W, Rinneberg H. 2001. MR-Thermometrie eines HF-geheizten körpergroßen Phantoms zur Simulation einer Tiefenhyperthermie. 4th Annual Meeting of the German Chapter of the ISMRM, Zurich, http://www.mr.ethz.ch/ismrm01/ praesentation.html.

23. Descorps M, Blondet P, Reutenauer H, Albrand JP, Remy C. 1985. An inductively coupled, series-tuned NMR probe. J Magn Reson 65:100-109.

24. Hoult DI, Lauterbur PC. 1979. The sensitivity of the zeugmatographic experiment involving human samples. J Magn Reson 34:425-433.

25. Gadian DG. 1979. Radiofrequency losses in NMR experiments on electrically conducting samples. J Magn Reson 34:449-455. 
26. Molière T. 1999. Feldstärkemessungen leicht gemacht (1). CQ DL—Das Amateurfunkmagazin 6/1999:484486.

27. Chen CN, Sank VJ, Cohen SM, Hoult DI. 1986. The field dependence of NMR imaging I. Laboratory assessment of signal-to-noise ratio and power deposition. Magn Reson Med 3:722-729.

28. Ǔgurbil K, Garwood M, Ellermann J, Hendrich K, Hinke R, Hu X, Kim SG, Menon R, Merkle H, Ogawa S, Salmi R. 1993. Imaging at high magnetic fields: Initial experiences at 4 T. Magn Reson Quart 9:259277.

29. Lee JH, Garwood M, Menon R, Adriany G, Andersen P, Truwit CL, Ǔgurbil K. 1995. High contrast and fast three-dimensional magnetic resonance imaging at high fields. Magn Reson Med 34:308-312.

30. Stollberger R, Wach P. 1996. Imaging of the active $B_{1}$ field in vivo. Magn Reson Med 35:246-251.

31. Mansfield P, Morris PG. 1982. NMR imaging in biomedicine. In: Waugh JS, editor. Advances in Magnetic Resonance, Supplement 2. New York: Academic Press. p 1-354.

32. Ogawa S, Lee TM, Kay AR, Tank DW. 1990. Brain magnetic resonance imaging with contrast depending on blood oxygenation. Proc Natl Acad Sci USA 87: 9868-9872.

33. Norris DG. 2000. Reduced power multislice MDEFT imaging. J Magn Reson Imag 11:445-451.

34. Hennig J, Nauerth A, Friedburg H. 1986. RARE imaging: A fast imaging method for clinical MR. Magn Reson Med 3:823-833.

35. Mansfield P. 1977. Multi-planar image formation using NMR spin echoes. J Phys C 10:L55-L58.

36. Stroop J. 1935. Studies of interference in serial verbal reactions. J Exp Psychol 18:643-662.

37. Zysset S, Müller K, Lohmann G, von Cramon DY. 2001. Color-word matching stroop task: Separating interference and response conflict. NeuroImage 13:2936.

38. Norris DG, Zysset S, Mildner T, Wiggins CJ. 2002. An investigation of the value of spin-echo-based fMRI using a Stroop color-word matching task and EPI at 3 T. NeuroImage 15:719-726.

39. Lohmann G, Müller K, Bosch V, Mentzel H, Hessler S, Chen L, Zysset S, von Cramon DY. 2001. LIPSIA: A new software system for the evaluation of functional magnetic resonance images of the human brain. Comput Med Imag Graph 25:449-457. 